\title{
Vortex Dynamics in Ferromagnetic/Superconducting Bilayers
}

\author{
M.Z. Cieplak, Z. Adamus \\ Institute of Physics, Polish Academy of Sciences \\ al. Lotników 32/46, 02-668 Warsaw, Poland \\ M. KOŃCZYKOWSKI \\ Laboratoire des Solides Irradiés, CEA/DSM/DRECAM and CNRS-UMR 7642 \\ Ecole Polytechnique, 91128 Palaiseau Cedex, France

\section{L.Y. ZHU AND C.L. CHIEN} \\ Department of Physics and Astronomy, The Johns Hopkins University \\ Baltimore, Md 21218, USA \\ The dependence of vortex dynamics on the geometry of magnetic do- \\ main pattern is studied in the superconducting/ferromagnetic bilayers, in \\ which niobium is a superconductor, and $\mathrm{Co} / \mathrm{Pt}$ multilayer with perpendicu- \\ lar magnetic anisotropy serves as a ferrromagnetic layer. Magnetic domain \\ patterns with different density of domains per surface area and different do- \\ main size, $w$, are obtained for $\mathrm{Co} / \mathrm{Pt}$ with different thickness of $\mathrm{Pt}$. The \\ dense patterns of domains with the size comparable to the magnetic pen- \\ etration depth $(w \geq \lambda)$ produce large vortex pinning and smooth vortex \\ penetration, while less dense patterns with larger domains $(w \gg \lambda)$ enhance \\ pinning less effectively and result in flux jumps during flux motion.
}

PACS numbers: 74.25.Ha, 74.25.Qt, 74.78.Db, 74.78.Fk

\section{Introduction}

One of the most important parameters for application of superconducting films in devices is the critical current density, which may be enhanced by artificial vortex pinning centers. A special type of pinning, called magnetic pinning (MP), occurs in ferromagnetic/superconducting bilayers (FSB), which are the structures consisting of thin superconducting $(\mathrm{S})$ and ferromagnetic $(\mathrm{F})$ films, with the thin insulating layer in-between to eliminate proximity effect $[1,2]$. Below the superconducting transition temperature, $T_{\mathrm{c}}$, when the external magnetic field, $H$, 
penetrates the $\mathrm{S}$ layer in the form of a vortex lattice, any magnetic texture which exists in the $\mathrm{F}$ layer interacts with the magnetic field generated by the vortex. This interaction creates an effective pinning potential, $U=-\boldsymbol{h}_{\mathrm{v}} \cdot \boldsymbol{m}$, which is asymmetric with respect to the alignment between $\boldsymbol{h}_{\mathrm{v}}$ and $\boldsymbol{m}\left(\boldsymbol{h}_{\mathrm{v}}\right.$ is the field of the vortex, and $\boldsymbol{m}$ is the magnetic moment of the texture) [1,2]. The domain structure in FSB's may be reversibly defined and erased allowing easy modulation of pinning. While there are many experiments which confirm the existence of the MP, only few of them attempt to correlate the vortex dynamics in the FSB's with the geometry of the magnetic domain patterns [3-10].

Recently, we have studied FSB's built of niobium as an S layer, and $\mathrm{Co} / \mathrm{Pt}$ superlattice with perpendicular magnetic anisotropy (PMA) as an F-layer. We have shown that the enhancement of pinning induced by the MP is the largest in the final stage of the magnetic reversal process of $\mathrm{Co} / \mathrm{Pt}$, when the domain patterns contain maze of the residual uninverted (RU) domains immersed in the inverted background $[4-6,9,10]$. However, so far it is unclear what is exactly the role of domain pattern geometry on the pinning. The only theoretical prediction states that the pinning is large when $\lambda \ll w$, where $\lambda$ is the magnetic penetration depth, and $w$ is the width of the magnetic domains $[1,11]$. In the present study we investigate the effect of different domain widths and density on the pinning enhancement and on the dynamics of flux penetration.

\section{Domain patterns}

The FSB's are grown using the high-vacuum magnetron sputtering system on the Si substrate at room temperature. To create two different FSB's with geometrically different domain patterns we use $[\mathrm{Co}(0.6 \mathrm{~nm}) / \operatorname{Pt}(t)] \times 8$ superlattice with two different thicknesses of platinum, $t=0.3 \mathrm{~nm}$ and $t=1.4 \mathrm{~nm}$. We call these samples Pt3 and Pt14, respectively. In both FSB's the F layers are covered by an amorphous, $10 \mathrm{~nm}$ thick Si film (to avoid proximity effect). Finally, Nb film, $78 \mathrm{~nm}$ thick, is grown on the top, with $T_{\mathrm{c}}=8.5 \mathrm{~K}$, as measured by standard magnetometry.

Figure 1a shows hysteresis loops for two FSB's measured above $T_{\mathrm{c}}(T=$ $10 \mathrm{~K}$ ) using anomalous Hall effect (AHE). To describe the state of the magnetic reversal we define parameter $s=\frac{1}{2}\left(M / M_{\mathrm{s}}+1\right)$, where $M$ is the magnetization, and $M_{\mathrm{s}}$ is $M$ at saturation. The loops have rectangular shape characteristic of films with PMA, with a sudden drop of magnetization when the inverted domains nucleate, followed by the long "tails" caused by the RU domains, as discussed previously $[4-6,9,10]$. The coercive fields are 640 Oe and 200 Oe for Pt3 and Pt14, respectively. The decrease in the coercive field with the increase in $t$ results from smaller coupling between Co layers in the $\mathrm{Co} / \mathrm{Pt}$ superlattice [12].

Figure 1b shows patterns imaged by magnetic force microscopy (MFM) for two FSB's. Both images are obtained after magnetizing the samples to the "tail" of the hysteresis loop (as shown by arrows in Fig. 1a), and then removing the field. 


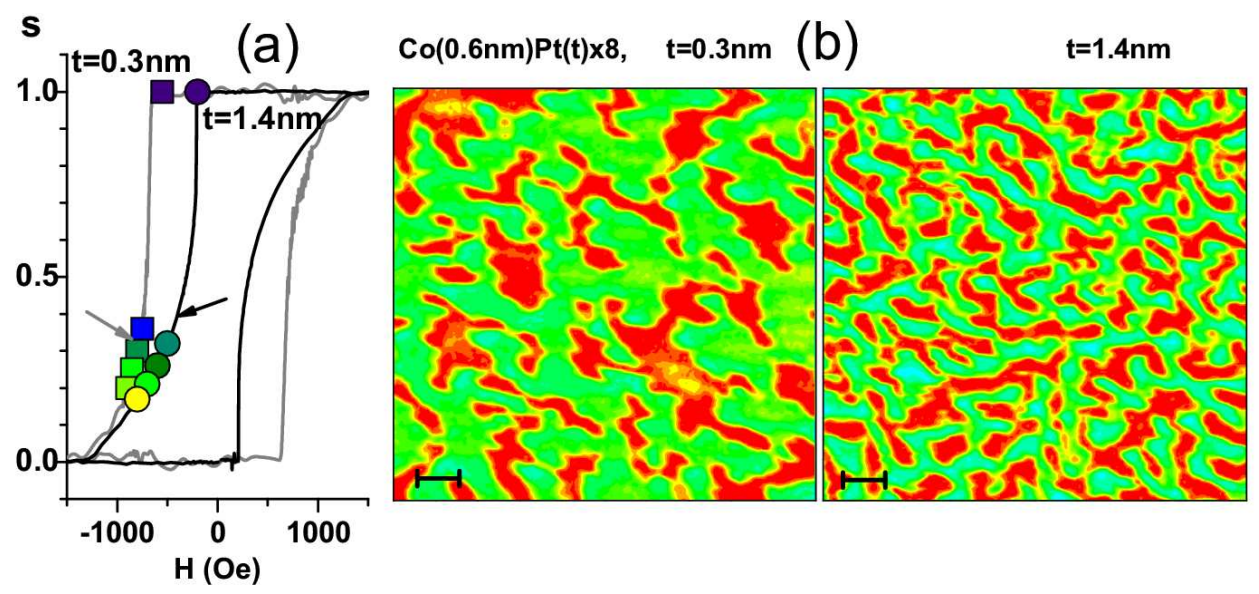

Fig. 1. (a) Hysteresis loops measured by AHE for Pt3 and Pt14 samples at $T=10 \mathrm{~K}$. Arrows and full points (circles and squares) indicate, respectively, the $s$-values at which MFM images are taken, and the $s$-values at which flux pinning is studied at $T<T_{\mathrm{c}}$. (b) MFM images $(10 \times 10 \mu \mathrm{m})$ taken at $T=300 \mathrm{~K}$ for Pt3 $(s \approx 0.35)$ and Pt14 sample $(s \approx 0.42)$. The bar shown at the bottom of images is $1 \mu \mathrm{m}$ long.

The resulting remnant domain patterns are stable, and consist of RU domains (dark) in the inverted (bright) background. The RU domains for Pt14 sample are relatively uniform in shape, narrow, with $w$ between 300 and $600 \mathrm{~nm}$. The distances between domains are small, only slightly larger than $w$. The pattern for Pt3 contains some amount of similar narrow domains, but, in addition, it contains much larger domains as well, with $w$ reaching 1000 to $1500 \mathrm{~nm}$. The distances between domains are large, of the order of the size of large domains. The increase in the domain size with decreasing thickness of the ultrathin ferromagnetic films is favorable energetically [13]. In the case of the $\mathrm{Co} / \mathrm{Pt}$ and similar multilayer systems experiments find nonmonotonic $w(d)$-dependence $(d$ is the total thickness of the multilayer), with the small- $d$ limit in which $w(d)$ is a decreasing function, and large- $d$ limit in which $w(d)$ increases $[14,15]$. In our experiment both samples are in a small- $d$ limit.

\section{Flux pinning}

The flux pinning is studied on samples cut into $240 \mu \mathrm{m}$ wide strips, with the set of 10 collinear miniature Hall sensors, of the area $5 \times 5 \mu \mathrm{m}^{2}$ each, placed across the strip, $20 \mu \mathrm{m}$ apart. We focus on the sensor nearby the middle of the sample. The experiment proceeds as follows. First, the RU domain patterns are defined at $T>T_{\mathrm{c}}$ by magnetizing the samples to various $s$-values (indicated by full points in Fig. 1a), and then removing the field. The process shown in Fig. 1a creates positive RU domains in the negative background. Next, the sample is cooled 
just below $T_{\mathrm{c}}$, and the local magnetic induction $B$ is measured for the external magnetic field $H$ between +90 Oe and -90 Oe.

Figure 2 shows the local magnetic field (equivalent to the magnetization) $H_{\text {loc }}=B-H$, plotted for two FSB's for $T=7 \mathrm{~K}$, and for several $s$-values. The loops measured for uniformly saturated F-layers ( $s=1$, RU domains absent), are approximately symmetric with respect to the $H$-sign. On the other hand, the loops measured for $s<0.5$, i.e. for F-layers magnetized to the "tail", are all asymmetric, with the width strongly enhanced for positive $H$. This indicates that the enhancement of pinning is caused by positive RU domains, in agreement with the asymmetric MP potential described in Sect. 1 [1, 2]. The enhancement, estimated as a ratio of the peak-to-peak hysteresis width for $s<0.5$, to the width for $s=1$, reaches about 3.3 for Pt3 sample, and about 5.7 for Pt14. We conclude that the pattern with smaller domains (Pt14) induces stronger MP, by a factor of about 1.7 .

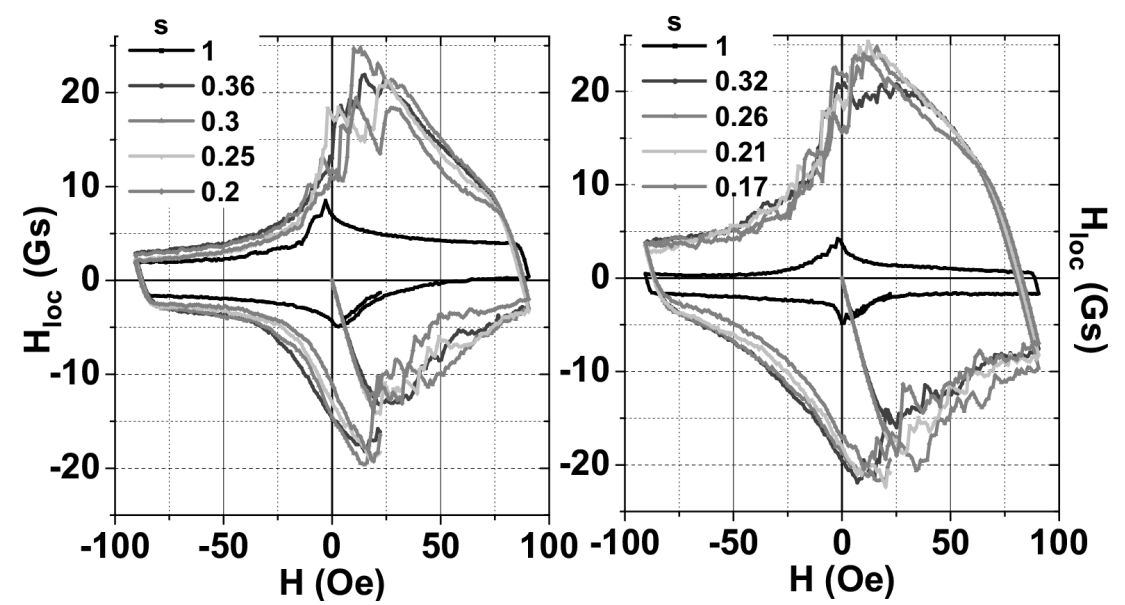

Fig. 2. Hysteresis loops taken at $T=7 \mathrm{~K}$ for several $s$ values for Pt3 (left) and Pt14 (right) samples. The loops for $s=1$ (F layer at saturation) are shown as reference.

We have estimated previously that the magnitude of the penetration depth, $\lambda(0)$, in our $\mathrm{Nb}$ layers is close to $95 \mathrm{~nm}$ [5]. Using the Ginzburg-Landau formula we estimate that at the temperature of our experiment, $T / T_{\mathrm{c}} \approx 0.88, \lambda$ becomes close to $200 \mathrm{~nm}$. Therefore, small domains $(w \approx 300 \mathrm{~nm})$ have the size just slightly larger than $\lambda$. It is very likely that they create strong pinning centers for vortices. The pinning should be particularly efficient in case of the pattern with uniform distribution of such strong pinning centers, and this is the case of Pt14 sample. More disordered pinning landscape is created in case of Pt3 sample, when domains are of various sizes. In particular, small, strongly pinning domains are in this case less abundant, which is probably partially responsible for weaker pinning enhancement. 
In addition, we observe that the shapes of the loops measured for two FSB's are different (Fig. 2). In case of Pt3 sample sudden jumps are seen in the top part of the loop. Moreover, the $H_{\text {loc }}$ drops to the value comparable to the $s=1$ loop when the external field changes polarity from positive to negative. This indicates strong expulsion of flux when the vortex field becomes antiparallel to the magnetic moment of the RU domains. In case of Pt14 sample the jumps are much smaller, and the expulsion of flux proceeds much more slowly. In fact, some enhancement of pinning persists even for large negative $H$, although it is much smaller than the enhancement for positive $H$.

These observations may be understood based on the MFM images. The disordered pinning landscape in Pt3 sample contributes to the strong pinning in some areas of the vortex lattice, and much weaker pinning in other areas, resulting in a sudden unpinning events of large portions of flux during the flux entry and flux expulsion (flux jumps). In addition, there are large inverted areas between RU domains, which repeal vortices with opposite polarity when the magnetic field polarity changes, and this results in an abrupt decrease in the vortex pinning in the vicinity of $H=0$. In the Pt14 sample the pinning landscape is more uniform, with larger density of smaller domains, which promotes smoother flux entry and exit. Moreover, the areas between RU domains are small, so that they themselves may behave as pinning centers for the flux of the opposite polarity, contributing to the enhancement of pinning which persists for negative $H$.

\section{Conclusion}

We have studied the influence of domain pattern geometry on the vortex dynamics in the $\mathrm{Nb} /(\mathrm{Co}, \mathrm{Pt})$ bilayers. Different magnetic domain patterns are obtained using $\mathrm{Co} / \mathrm{Pt}$ superlattice with different $\mathrm{Pt}$ thickness. We find that the large magnetic pinning is induced by the pattern with large density of small domains, of the size $w$ comparable to the penetration depth, $\lambda$. This type of domain pattern results in smooth penetration of flux. Increasing the domain size $(w \gg \lambda)$ and the distances between domains results in a decrease in pinning, and the appearance of sudden flux unpinning events during the flux entry and exit.

\section{Acknowledgments}

This work was supported by the MNiSW grants N202 058 32/1202 and N202 019 31/0727, by the NSF grants DMR05-20491 and DMR04-03849, and by the French government scholarship for Z. Adamus.

\section{References}

[1] I.F. Lyuksyutov, V.L. Pokrovsky, Adv. Phys. 54, 67 (2004).

[2] M.V. Milošević, S.V. Yampolskii, F.M. Peeters, Phys. Rev. B 66, 174519 (2002).

[3] M. Lange, M.J. Bael, V.V. Moshchalkov, Y. Bruynseraede, Appl. Phys. Lett. 81, $322(2002)$ 
[4] M.Z. Cieplak, Z. Adamus, M. Konczykowski, X.M. Cheng, A. Byczuk, A. Abal'oshev, H. Sang, C.L. Chien, Acta Phys. Pol. A 106, 693 (2004).

[5] M.Z. Cieplak, X.M. Cheng, C.L. Chien, H. Sang, J. Appl. Phys. 97, 026105 (2005).

[6] M.Z. Cieplak, Z. Adamus, A. Abal'oshev, I. Abal'osheva, M. Berkowski, X.M. Cheng, H. Sang, C.L. Chien, Phys. Status Solidi C 2, 1650 (2005).

[7] F. Laviano, L. Gozzelino, E. Mezzetti, P. Przyslupski, A. Tsarou, A. Wisniewski, Appl. Phys. Lett. 86, 152501 (2005).

[8] L. Gozzelino, F. Laviano, P. Przyslupski, A. Tsarou, A. Wisniewski, D. Botta, R. Gerbaldo, G. Ghigo, Supercond. Sci. Technol. 19, S50 (2006).

[9] Z. Adamus, M.Z. Cieplak, A. Abal'oshev, M. Berkowski, M. Konczykowski, X.M. Cheng, L.Y. Zhu, C.L. Chien, Acta Phys. Pol. A 109, 451 (2006).

[10] Z. Adamus, M.Z. Cieplak, A. Abal'oshev, M. Berkowski, M. Konczykowski, X.M. Cheng, L.Y. Zhu, C.L. Chien, Acta Phys. Pol. A 111, 95 (2007).

[11] L.N. Bulaevskii, E.M. Chudnovsky, M.P. Maley, Appl. Phys. Lett. 76, 2594 (2000).

[12] J.W. Kneopper, F.Y. Yang, Phys. Rev. B 71, 224403 (2005).

[13] C. Kittel, Phys. Rev. 70, 965 (1946).

[14] G. Bochi, H.J. Hug, D.I. Paul, B. Stiefel, A. Moser, I. Parashikov, H.-J. Güntherodt, R.C. O'Handley, Phys. Rev. Lett. 75, 1839 (1995).

[15] O. Hellwig, A. Berger, E.E. Fullerton, J. Magn. Magn. Mater. 290-291, 1 (2005). 